\title{
A IMPORTÂNCIA DAS REVISTAS QUÍMICA NOVA E JOURNAL OF THE BRAZILIAN CHEMICAL SOCIETY NO CRESCIMENTO DA ÁREA DE QUÍMICA NO BRASIL ${ }^{\#}$
}

\author{
Susana I. Córdoba de Torresi e Vera L. Pardini \\ Departamento de Química Fundamental, Instituto de Química, Universidade de São Paulo, CP 26077, 05513-970 São Paulo - \\ SP, Brasil \\ Luiz C. Dias \\ Instituto de Química, Universidade Estadual de Campinas, CP 6154, 13084-971 Campinas - SP, Brasil \\ Angelo C. Pinto \\ Instituto de Química, Universidade Federal do Rio de Janeiro, CT, Bl. A, Cidade Universitária, 21945-970 Rio de Janeiro - RJ, \\ Brasil \\ Jailson B. de Andrade* \\ Instituto de Química, Universidade Federal da Bahia, Departamento de Química Geral e Inorgânica, Campus Universitário de \\ Ondina, 40170-290 Salvador - BA, Brasil \\ Maria Elizabeth A. Magalhães e Pricila Esteves de Almeida Gil \\ Sociedade Brasileira de Química, CP 26037, 05513-970 São Paulo - SP, Brasil
}

\begin{abstract}
THE IMPORTANCE OF QUIMICA NOVA AND JOURNAL OF THE BRAZILIAN CHEMICAL SOCIETY FOR THE DEVELOPMENT OF CHEMISTRY IN BRAZIL. Quimica Nova and the Journal of the Brazilian Chemical Society are two examples of successful initiatives taken by the Brazilian Chemical Society (SBQ - Sociedade Brasileira de Química), and may serve as models for the scientific societies of developing countries. Pillars of the SBQ, these two periodicals are undeniable demonstrations that idealism, utopia and dignity are the essential ingredients for transforming dreams into reality. Few believed that the Brazilian chemical community would one day have, as it does today, two scientific research periodicals indexed in the principal international data banks.
\end{abstract}

Keywords: SciELO; Web of Science; Impact Factor.

\section{INTRODUÇÃO}

A Sociedade Brasileira de Química publica regularmente três periódicos (Química Nova, Journal of the Brazilian Chemical Society e Química Nova na Escola) e um Boletim Eletrônico. O objetivo deste artigo é traçar um panorama atual e a trajetória das publicações da Sociedade Brasileira de Química (Química Nova e Journal of the Brazilian Chemical Society) desde o lançamento, em 1978, da revista Química Nova - um dos marcos históricos da SBQ, mostrando a evolução destas duas revistas e os desafios que são enfrentados diariamente pelos editores destas publicações científicas editadas no País.

\section{QUÍMICA NOVA}

A idéia de uma revista de Química em língua portuguesa precede a da criação da Sociedade Brasileira de Química, porque não seria possível o lançamento de Química Nova em janeiro de 1978, cinco meses após a criação da SBQ, se a concepção da revista não estivesse amadurecida pelo seu criador, Prof. Eduardo Peixoto. Cabe relembrar um parágrafo do editorial, escrito pelo Prof. Fernando Galembeck, na Edição Comemorativa dos 10 Anos da SBQ “Química Nova é, hoje, a mais palpável mudança na Química brasileira decorrente da fundação da SBQ. É uma revista procurada, lida e muito usada para transmitir idéias, resultados de pesquisa, experi-

\footnotetext{
\# Este artigo é dedicado à Helena Ferraz cujo desprendimento e empenho em relação à Química Nova (da qual foi editora de 1989 a 1994, assessora e autora) é exemplo para as gerações futuras. Desde o início acreditou no sucesso do $J B C S$, prestigiando-o com o resultado de suas pesquisas. *e-mail: jailsong@ufba.br
}

ências de ensino, textos de difusão. Está nas Bibliotecas, nas estantes, com um aspecto mais colorido do que austero. É um documento vivo, não um repositório de letras sepultadas. É útil e fertilizadora"'.

Criada para ser o órgão oficial de divulgação da SBQ, Química Nova firmou-se ao longo dos anos como uma das revistas científicas brasileiras mais importantes, extrapolando as fronteiras nacionais, quando a partir de 1995 passou a ser indexada ao Web of Science ${ }^{2}$. Só cerca de duas dezenas de revistas, das milhares editadas no País, estão indexadas no Institute for Scientific Information (ISI).

Sem perder sua essência e, sobretudo, sua brasilidade, razão de seu sucesso, Química Nova é a $3^{\mathrm{a}}$ revista mais acessada no SciELO (sigla em inglês que significa Biblioteca Científica Eletrônica Online) com mais de 5 milhões de acessos (http://www.scielo.br). A coleção SciELO é uma das 10 fontes de informação mais acessadas por usuários do Google Scholar, ferramenta da Google especializada em pesquisa acadêmica ${ }^{3}$.

Além de ser uma biblioteca on-line de periódicos científicos, a SciELO adota uma metodologia inspirada naquela do Thomson Scientific, antigo Institute for Scientific Information (ISI). A base SciELO tem indicadores estatísticos de citação e impacto de revistas e artigos, e fornece, por exemplo, o número de visitas aos artigos de todas as revistas de sua coleção.

A Química Nova (http://quimicanova.sbq.org.br) publica artigos com resultados originais de pesquisa, trabalhos de revisão, de novos métodos ou técnicas, de educação, assuntos gerais e sobre história da Química.

A Tabela 1 relaciona o número de trabalhos publicados na revista no período 2000-2006, por modalidade. Como pode ser visto, predomina o número de artigos. Como não mais serão publicados artigos de divulgação, espera-se que aumente a publicação de artigos de revisão, que se mantém praticamente dentro da mesma faixa desde 2002. 
Tabela 1. Distribuição do tipo de contribuição, por modalidade, no período 2000-2006

\begin{tabular}{|c|c|c|c|c|c|c|c|}
\hline Trabalhos & 2000 & 2001 & 2002 & $\begin{array}{l}\text { Ano } \\
2003\end{array}$ & 2004 & 2005 & 2006 \\
\hline Artigos & 53 & 60 & 77 & 65 & 77 & 96 & 113 \\
\hline Revisão & 21 & 23 & 29 & 33 & 33 & 26 & 36 \\
\hline Divulgação & 30 & 23 & $33+12$ (esp.) & 17 & 20 & 29 & 32 \\
\hline Notas Técnicas & 8 & 9 & 10 & 18 & 13 & 15 & 19 \\
\hline Educação & 13 & 12 & 14 & 16 & 12 & 15 & 13 \\
\hline História da Química & 5 & 3 & 3 & 1 & 8 & 2 & 7 \\
\hline
\end{tabular}

A Figura 1 mostra o número de acessos dos 10 artigos de Química Nova mais consultados no SciELO. Apesar do número de consultas não ser um indicador adequado de qualidade e de impacto, este número é importante para mostrar temas de interesse mais geral para aqueles que os procuram na internet. Os temas dos 10 artigos mais acessados de Química Nova envolvem plantas medicinais ${ }^{5,6}$, desafios e perspectivas de produtos naturais ${ }^{7}$, biorremediação ${ }^{8}$, biotransformação de óleos e gorduras ${ }^{9}$, biodiesel ${ }^{10,11}$, estrutura química e tabela periódica ${ }^{12}$, determinação de massa molar por crioscopia ${ }^{13}$ e medidas de $\mathrm{pH}$ usando corantes naturais ${ }^{14}$. A maioria destes artigos é de interesse geral e são dirigidos, principalmente, para estudantes de graduação, pós-graduação e professores de ensino superior.

A Tabela 2 traz os 10 trabalhos mais citados, segundo o Web of Science. Destes, 7 são revisões, 2 são divulgações e apenas um é artigo. Os dois primeiros têm o mesmo número de citações (34), sendo que a divulgação versa sobre Quimiometria e a revisão, sobre Polianilinas.

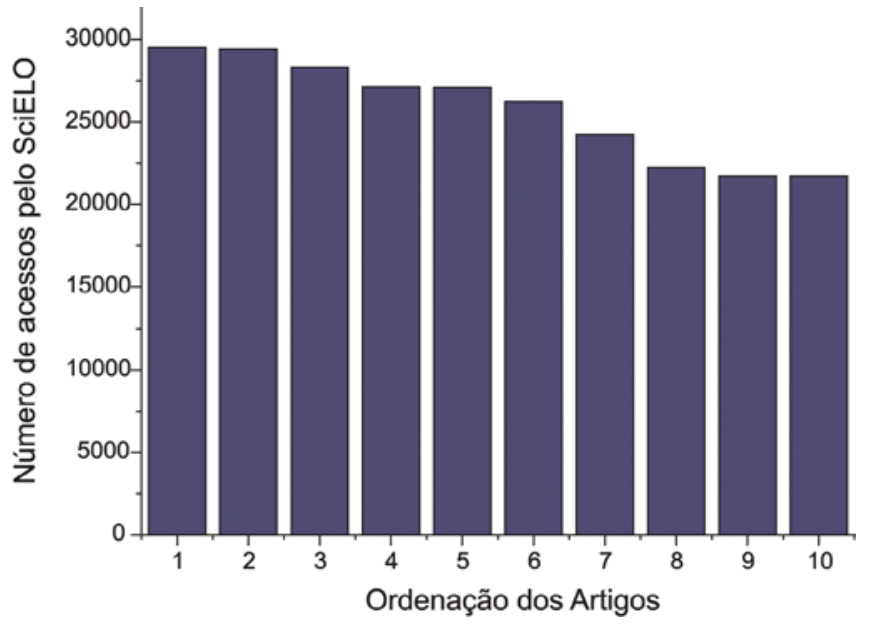

Figura 1. Ordem dos 10 artigos mais acessados na Química Nova via SciELO
As tabelas e gráficos que se encontram a seguir são ilustrativos das atividades e da evolução de Química Nova no período 2000-2006.

O Fator de Impacto ${ }^{25}$ de 0,72 alcançado, em 2006, por Química Nova é uma verdadeira façanha para uma revista escrita quase que integralmente em português, e reflete o grau de consolidação e de amadurecimento atingido pela Química brasileira.

A classificação de Química Nova como revista internacional A no sistema Qualis da CAPES pelos comitês de algumas áreas do conhecimento, como, por exemplo, Geociências e Ciência dos Alimentos, devido ao seu alto fator de impacto, fez com que ocorresse um aumento expressivo, a partir de 2005 dos trabalhos referentes a química ambiental e, ultimamente, de química de alimentos e de bebidas. Por outro lado, este crescimento foi acompanhado da diminuição de publicações na área de Química Analítica, tradicionalmente muito bem representada na Química Nova, devido, também, à migração de alguns químicos analíticos para a área de ambiental (Figura 2).

Na Tabela 3 e Figura 3 são apresentadas as publicações de cada região do Brasil e do exterior, por ano. Nota-se um ligeiro aumento da contribuição de pesquisadores do exterior em 2006, que pode ser atribuída à entrada em funcionamento do sistema de submissão on line, a partir de outubro de 2006.

Na Tabela 4 são mostradas as contribuições de cada estado, reunidos por regiões, no período 2000-2006. A classificação foi feita considerando só o autor correspondente de cada trabalho.

As Figuras 4, 5 e 6 complementam a Tabela 4, mostrando as publicações por estado das regiões Sudeste, Sul e Nordeste. Não foram considerados os estados que integram as regiões Norte e Centro-Oeste, devido ao reduzido número de contribuições. A região Sudeste $^{26}$ (Figura 4) continua sendo aquela com maior número de contribuições para Química Nova, com grande aumento das publicações de autores de São Paulo em 2006. Na região Sul (Figura 5) o número de artigos de autores do Paraná pela primeira vez, em 2006, ultrapassou a produção de autores dos estados do Rio Grande do Sul e de Santa Catarina. A Figura 6 indica que de 2000 a 2003 havia predominância da Bahia, mas nos últimos 3 anos nota-se um

Tabela 2. Os dez artigos mais citados em Química Nova, segundo Web of Science

\begin{tabular}{|c|c|c|c|c|}
\hline Artigo & $\begin{array}{l}\text { Química Nova } \\
\text { Ano, Volume, Pgs. }\end{array}$ & Autores & Categoria & № Citações \\
\hline 1 & $1999,22,724-731$ & Ferreira, M. M. C.; Antunes, A. M.; Melgo, M. S.; Volpe, P. L. O. & Divulgação & $34^{15}$ \\
\hline 2 & $\mathbf{1 9 9 6}, 19,388-399$ & Mattoso, L. H. C. & Revisão & $34^{16}$ \\
\hline 3 & $\mathbf{1 9 9 5}, 18,362-367$ & Moreira, J. C.; Barek, K. & Revisão & $33^{17}$ \\
\hline 4 & $\mathbf{2 0 0 0}, 23,71-78$ & Guaratini, C. C. I.; Zanoni, M. V. B. & Revisão & $27^{18}$ \\
\hline 5 & 1996, $19,497-519$ & Lopes,W. A.; de Andrade, J. B. & Revisão & $26^{19}$ \\
\hline 6 & $1999,22,246-253$ & Arakaki, L. N. H.; Airoldi, C. & Revisão & $25^{20}$ \\
\hline 7 & $\mathbf{2 0 0 0}, 23,98-101$ & Coelho, F.; Almeida, W. P. & Divulgação & $23^{21}$ \\
\hline 8 & $\mathbf{1 9 9 8}, 21,300-311$ & Crepaldi, E. L.; Valim, J. B. & Revisão & $23^{22}$ \\
\hline 9 & $\mathbf{2 0 0 0}, 23,310-312$ & Aleixo, P. C.; Nóbrega, J. A.; Santos Jr., D.; Muller, R. C. S. & Artigo & $22^{23}$ \\
\hline 10 & $\mathbf{2 0 0 0}, 23,204-215$ & Maia, D. J.; De Paoli, M-A.; Alves, O. L.; Zarbin, A. J. G.; das Neves, S. & Revisão & $21^{24}$ \\
\hline
\end{tabular}




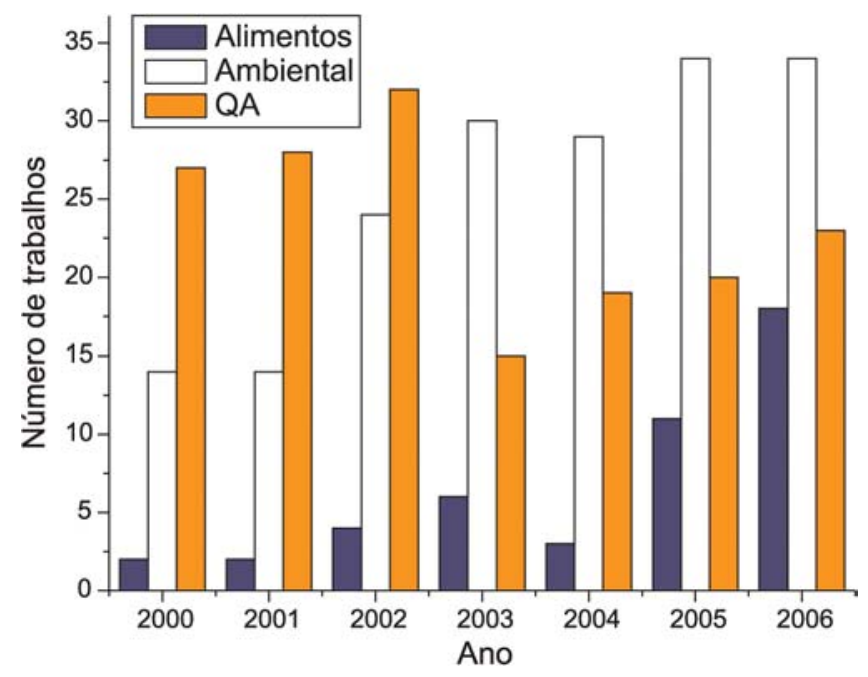

Figura 2. Número de trabalhos publicados nas áreas de Química Analítica (QA), Química Ambiental (AB) e Química de Bebidas e Alimentos (BA)

Tabela 3. Número de artigos publicados no período 2000-2006 por regiões do Brasil e exterior

\begin{tabular}{ccccccc}
\hline & Norte & Nordeste & Centro-Oeste & Sudeste & Sul & Ext \\
\hline 2000 & 3 & 5 & 1 & 99 & 15 & 8 \\
2001 & 1 & 8 & 3 & 95 & 21 & 6 \\
2002 & 2 & 17 & - & 135 & 22 & 6 \\
2003 & 3 & 13 & 5 & 98 & 29 & 6 \\
2004 & 1 & 18 & 2 & 117 & 19 & 9 \\
2005 & 2 & 21 & 2 & 123 & 30 & 9 \\
2006 & - & 20 & 4 & 151 & 34 & 13 \\
\hline
\end{tabular}

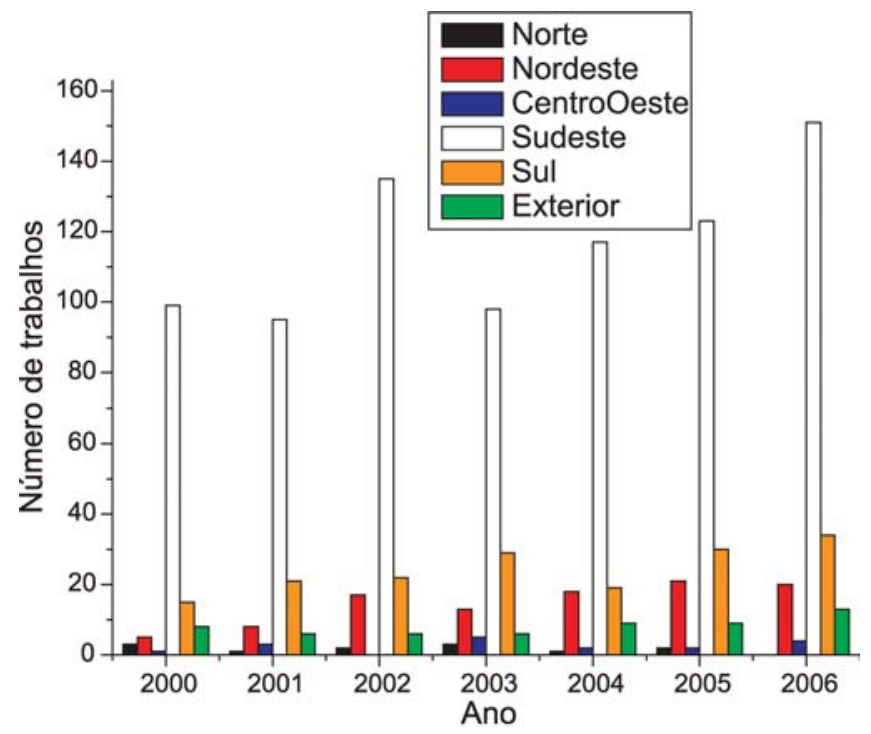

Figura 3. Artigos publicados no período 2000-2006 por regióes do Brasil e exterior

crescimento da região como um todo, destacando-se o Ceará. É importante salientar que o maior número de publicações provenientes de Pernambuco no período 2004-2006, quando comparado a 20002003 se deve à área de Alimentos e coincide com o interesse da área
Tabela 4. Número de artigos publicados por regiões e estados brasileiros no período 2000-2006

\begin{tabular}{|c|c|c|c|c|c|c|c|}
\hline & 2000 & 2001 & 2002 & 2003 & 2004 & 2005 & 2006 \\
\hline \multicolumn{8}{|l|}{ NORTE } \\
\hline $\mathrm{AM}$ & & 1 & & & 1 & & \\
\hline PA & & & & & & 1 & \\
\hline \multicolumn{8}{|l|}{ TO } \\
\hline RO & & & & & & 1 & \\
\hline $\mathrm{RR}$ & 3 & & 2 & 3 & & & \\
\hline \multicolumn{8}{|c|}{ NORDESTE } \\
\hline AL & & & 1 & & & & \\
\hline BA & 3 & 3 & 6 & 9 & 5 & 5 & 5 \\
\hline $\mathrm{CE}$ & & 1 & 4 & 2 & 1 & 2 & 5 \\
\hline MA & & & 3 & 1 & & 2 & \\
\hline RN & & 1 & 1 & & 1 & & 1 \\
\hline PB & 1 & 3 & 1 & & 1 & 1 & 4 \\
\hline PE & 1 & & & 1 & 7 & 6 & 3 \\
\hline PI & & & 1 & & 2 & 5 & 2 \\
\hline SE & & & & & 1 & & \\
\hline \multicolumn{8}{|c|}{ CENTRO-OESTE } \\
\hline MT & & 1 & & & & & \\
\hline MTS & & 1 & & 1 & & 1 & 1 \\
\hline GO & 1 & & & 1 & & & \\
\hline DF & & 1 & & 3 & 2 & 1 & 3 \\
\hline \multicolumn{8}{|c|}{$\begin{array}{l}\text { SUDESTE } \\
\text { SUDS }\end{array}$} \\
\hline SP & 71 & 60 & 91 & 66 & 69 & 84 & 108 \\
\hline MG & 11 & 12 & 15 & 14 & 13 & 23 & 20 \\
\hline RJ & 17 & 22 & 27 & 18 & 34 & 16 & 22 \\
\hline ES & & 1 & 2 & & 1 & & 1 \\
\hline \multicolumn{8}{|l|}{$\overline{\text { SUL }}$} \\
\hline PR & 4 & 7 & 7 & 9 & 5 & 11 & 16 \\
\hline $\mathrm{SC}$ & 6 & 6 & 9 & 6 & 4 & 2 & 8 \\
\hline $\mathrm{RS}$ & 5 & 8 & 6 & 14 & 10 & 17 & 10 \\
\hline
\end{tabular}

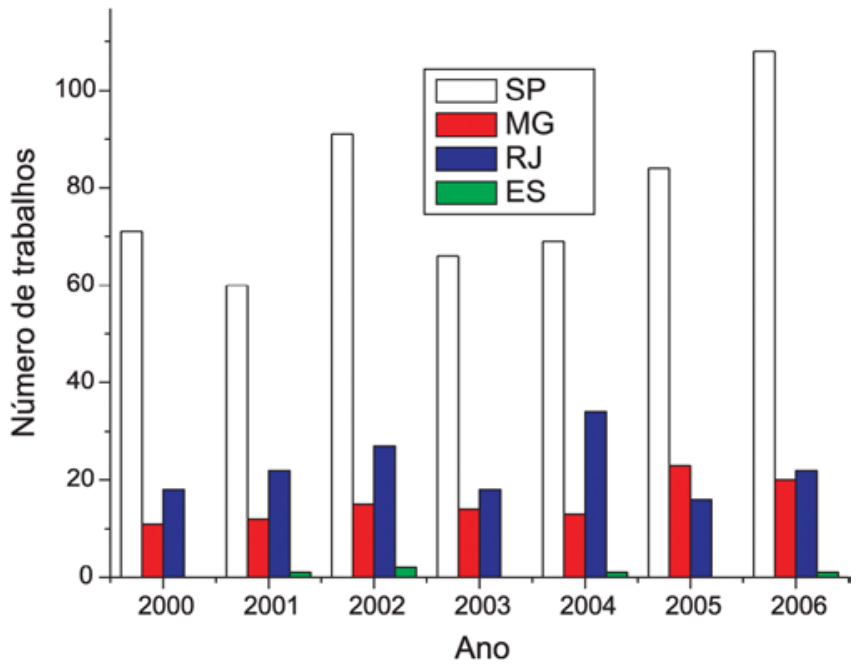

Figura 4. Distribuição de artigos publicados no período de 2000-2006 na Região Sudeste

em publicar em Química Nova, em virtude da classificação Qualis.

A Figura 7 mostra claramente o crescimento da revista, não só quanto à submissão mas também em relação ao número de trabalhos publicados, denotando o esforço dos Editores e assessores. Os 


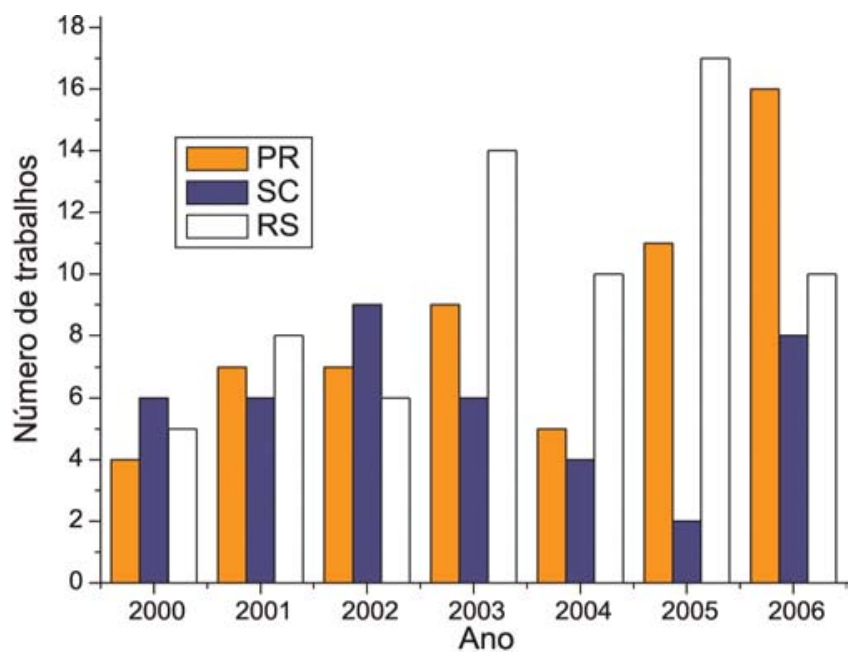

Figura 5. Distribuição de artigos publicados no período de 2000-2006 na Região Sul

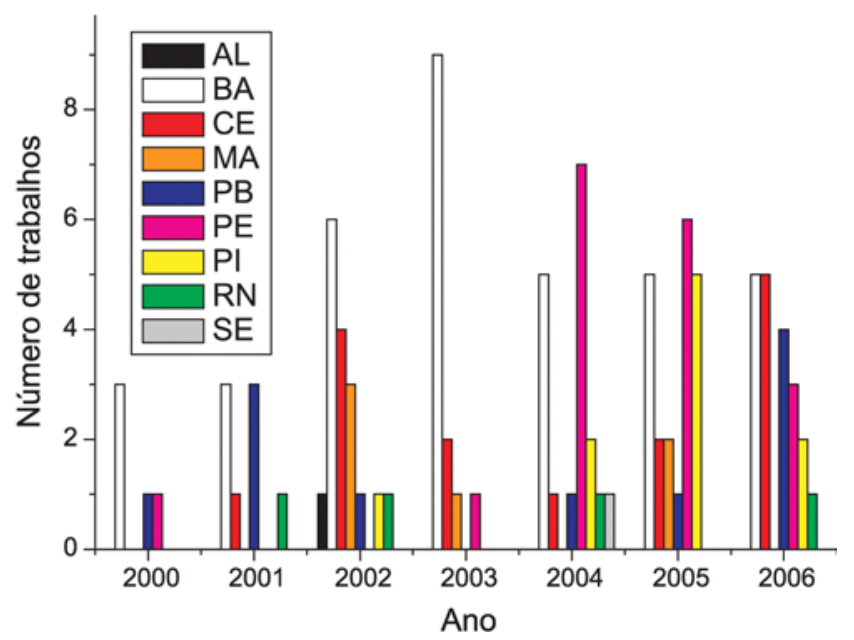

Figura 6. Distribuição de artigos publicados no período de 2000-2006 na Região Nordeste

dados referentes a 2007 levam em conta a submissão/aceitação até o mês de setembro, porém indicam uma evidente tendência de crescimento. Como já mencionado anteriormente, os dados de 2006 2007 estão relacionados com a implantação da submissão on line, bem como à classificação de Química Nova como Internacional B na área de Química, no Qualis/Capes, mas principalmente como Internacional $\mathrm{A}$ em áreas afins. Outros fatores que devem ser mencionados são a visibilidade que a revista alcançou com a indexação no SciELO e o aumento do Fator de Impacto nos últimos anos.

Na Figura 8, são mostrados os dados extraídos da base de dados ISI/Web of Science dos trabalhos publicados e número de citações recebidas em revistas indexadas na mesma base de dados. É claro que o número de citações cresceu mais rapidamente que o número de trabalhos, o que condiz com o Fator de Impacto crescente.

\section{JOURNAL OF THE BRAZILIAN CHEMICAL SOCIETY}

Em 1987, dez anos após a criação de Química Nova, no momento em que a Química no Brasil passava por um amplo processo de ampliação e reorganização com o Programa de Apoio ao Desenvolvimento Científico e Tecnológico (PADCT), sem dúvida um

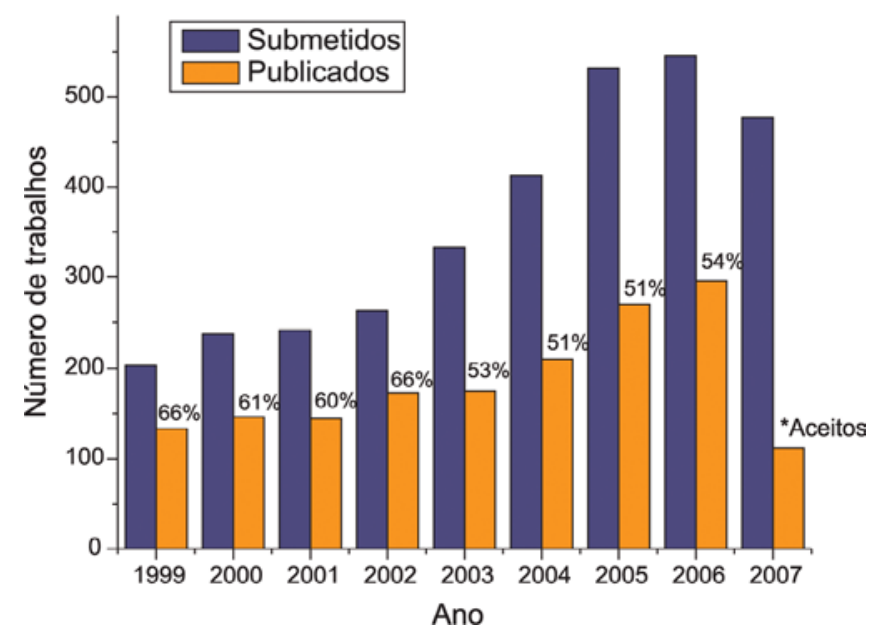

Figura 7. Distribuição de artigos submetidos e publicados no período de 1999 a 2006. Em 2007 são apresentados os submetidos e aceitos, até setembro

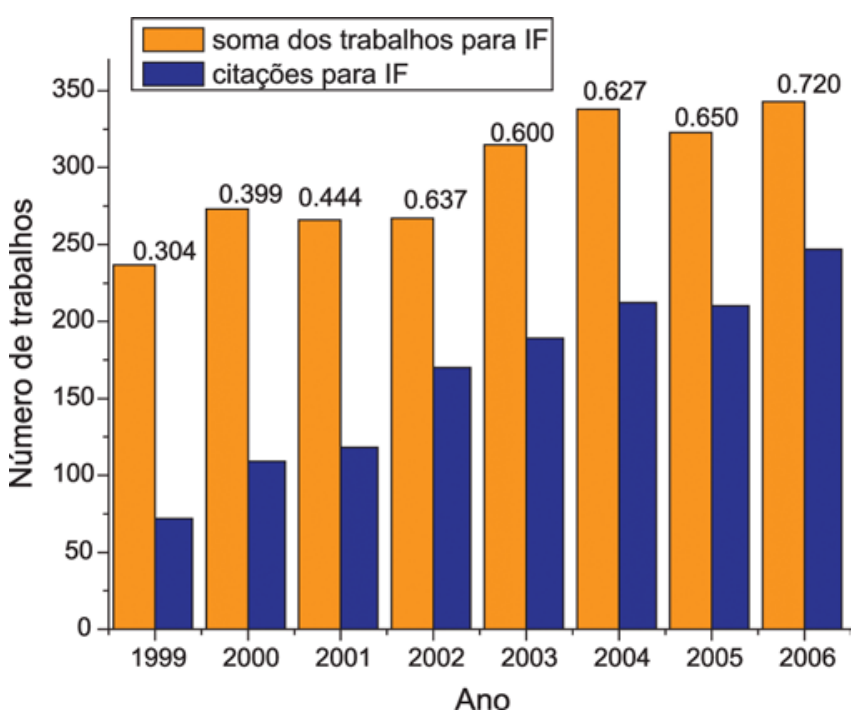

Figura 8. Total de trabalhos publicados (1995-2007) e total de citações computadas na base de dados ISI/Web of Science

dos Programas de maior importância da química brasileira e coresponsável pelo seu crescimento ${ }^{27,28}$, foi iniciado o processo de criação de um novo periódico da SBQ, com uma linha editorial distinta da de Química Nova, e em inglês ${ }^{29}$.

Em atendimento à chamada 10 do Edital QEQ 01/87, foi concebido o Journal of the Brazilian Chemical Society $(J B C S)^{29}$. O primeiro número do JBCS foi lançado em 1990, mais uma vez com o "visionário" Eduardo Peixoto como Editor-in-Chief.

O JBCS, além de ter sido um dos periódicos pioneiros de livre acesso na internet, [entrou no web of Science em 1995] foi a primeira revista científica brasileira a ultrapassar, em 2002, a barreira de fator de Impacto = 1 (Figura 9), que se mantém apesar do número de artigos publicados vir aumentando - 30\% em $2004^{30}$, 35\% em $2005^{31}$ e em 2006, ter quase que dobrado em relação a $2002^{32}$ (Figura 9). A revista firma-se, assim, como uma das mais importantes da área de Química das nações em desenvolvimento.

A Figura 10 mostra na coluna azul o número de citações, em cada ano, de artigos publicados nos dois anos anteriores, que contribuíram para o cálculo do fator de impacto. Mostra, também, na 
coluna laranja, a soma das publicações naquele ano utilizadas no denominador para cálculo do fator de impacto.

O ano de 2004 pode ser considerado um dos divisores de água do Journal of the Brazilian Chemical Society, quando foi implantado o sistema de submissão on-line de manuscritos e quando o Comitê da CAPES classificou o JBCS como Qualis 1A (Figura 11).

O aumento no número de submissões desde 2004 foi acompanhado de vertiginoso crescimento no número de artigos submetidos do exterior, que, em 2006, culminou com mais submissões do exterior que do Brasil, número que se mantém em 2007 (Figura 12).

O número de artigos submetidos do exterior pela primeira vez, em 2006, ultrapassou o número de artigos submetidos por autores do País, tendência esta que se acentua no ano de 2007. Por outro lado, o número de artigos publicados por pesquisadores brasileiros é maior (70\%) que de autores estrangeiros (Figura 12).

Desde fevereiro de 1998 até setembro de 2007, o número de acessos ao $J B C S$ pelo site do SciELO foi de aproximadamente 1 milhão.

O número de acessos ao JBCS pelo site do SciELO foi de aproximadamentre 230.000 em 2006, marca esta já ultrapassada em setembro de 2007, com mais de 275.000 acessos (Figura 13).

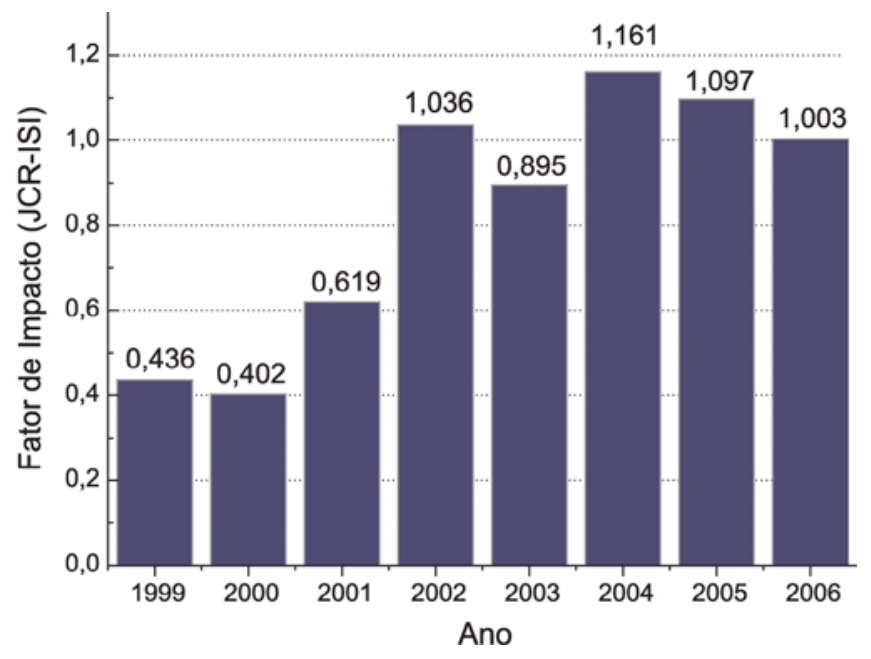

Figura 9. Fator de Impacto do JBCS no período de 2002-2006

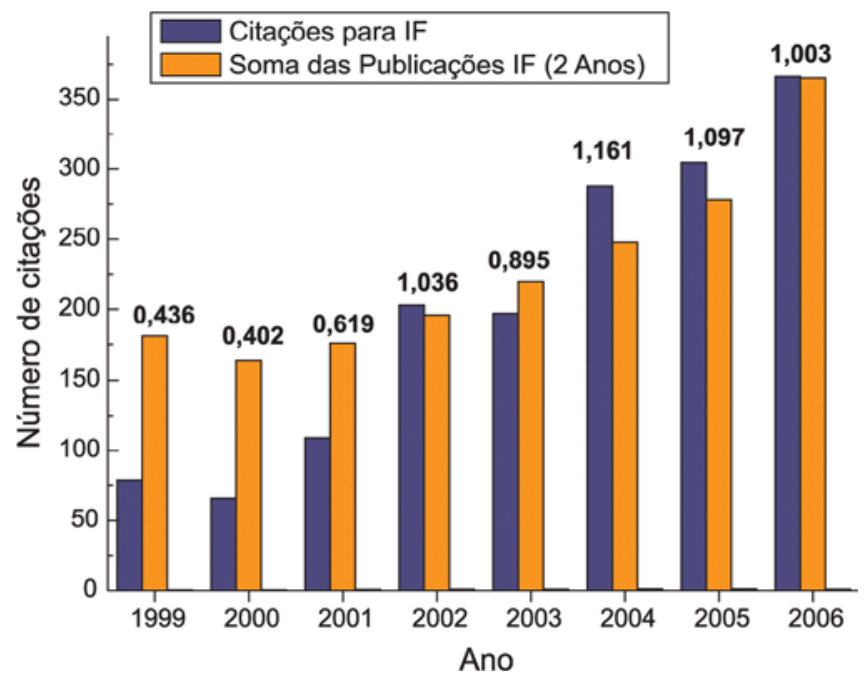

Figura 10. Soma das publicações e número de citações para cálculo do IF (1999-2006)
Entre os 10 artigos mais acessados no SciELO, no período de janeiro de 2004 a agosto de 2007, 7 são artigos de revisão e 3 destes estão entre os 10 mais citados do JBCS no Web of Science. Os 2 artigos mais acessados versam sobre biodiese ${ }^{33,34}$ devido ao interesse atual do tema.

As Figuras 14 e 15 mostram a origem por estados, de artigos publicados no $J B C S$, no intervalo de 10 anos. As flutuações foram poucas no período. As mais destacadas foram a participação do Rio Grande do Sul que dobrou, o crescimento do percentual de São Paulo e a retração de Minas Gerais e do Rio de Janeiro. A região sudeste foi responsável, em 2006, por $60 \%$ dos artigos publicados, mais da metade destes sendo do estado de São Paulo.

A Figura 15 mostra a distribuição dos artigos publicados nos últimos dez anos (1997-2006) revelando um saudável equilíbrio entre as subáreas de Química Analítica, Química Inorgânica, Química Orgânica, Química de Produtos Naturais, Eletroquímica e Físico-Química.

A Tabela 5 relaciona os artigos publicados no $J B C S$ com número de citações no Web of Science igual ou superior a 2030,35-54. Dos 21 artigos relacionados, 9 são artigos de revisão, 3 dos quais foram publicados em números especiais. Os 4 artigos mais citados são todos de revisão e têm 73 ou mais citações. A publicação mais cita-

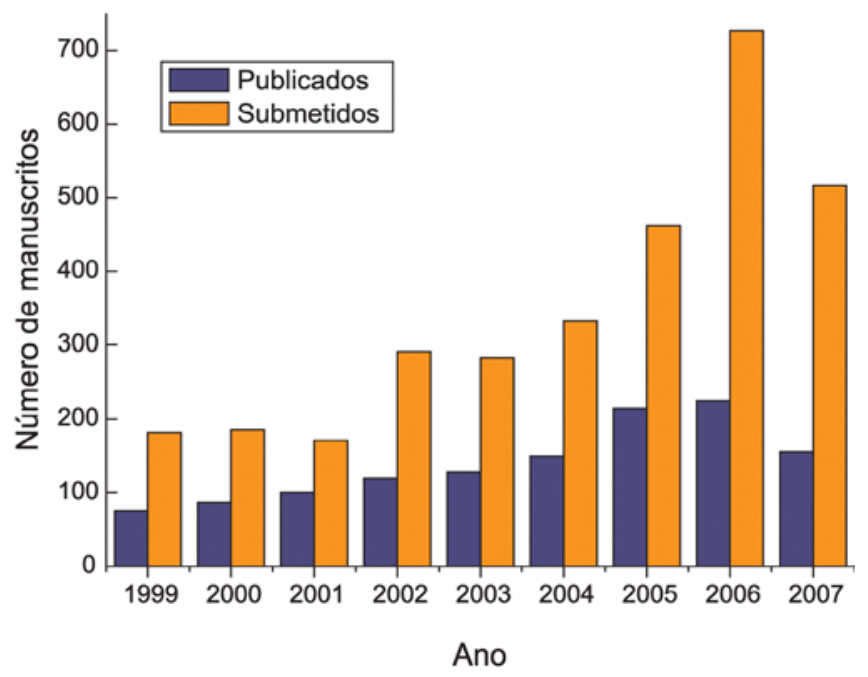

Figura 11. Número de manuscritos submetidos e publicados (1999-2006, 2007 em andamento)

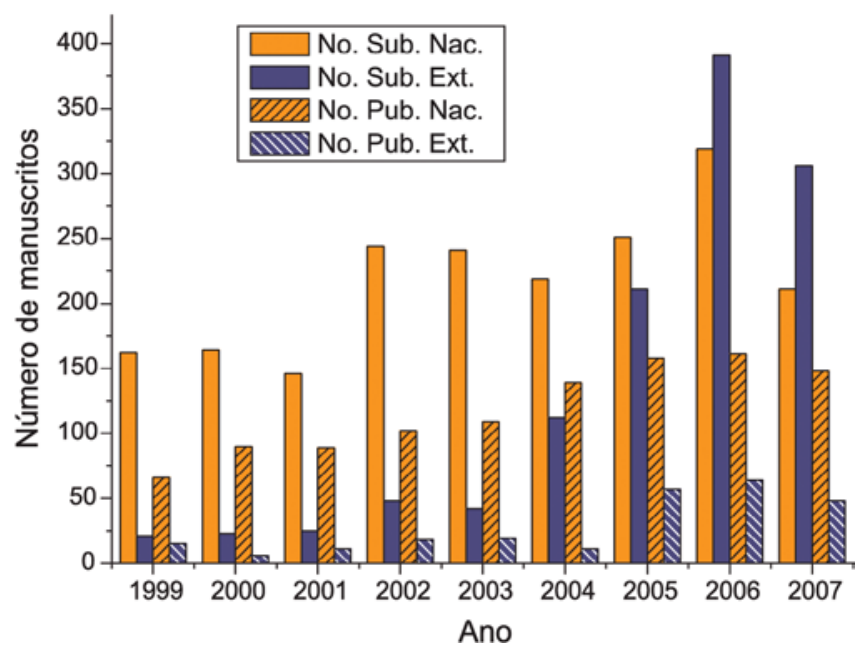

Figura 12. Número de manuscritos nacionais e do exterior, submetidos $e$ publicados (1999-2006, 2007 em andamento) 


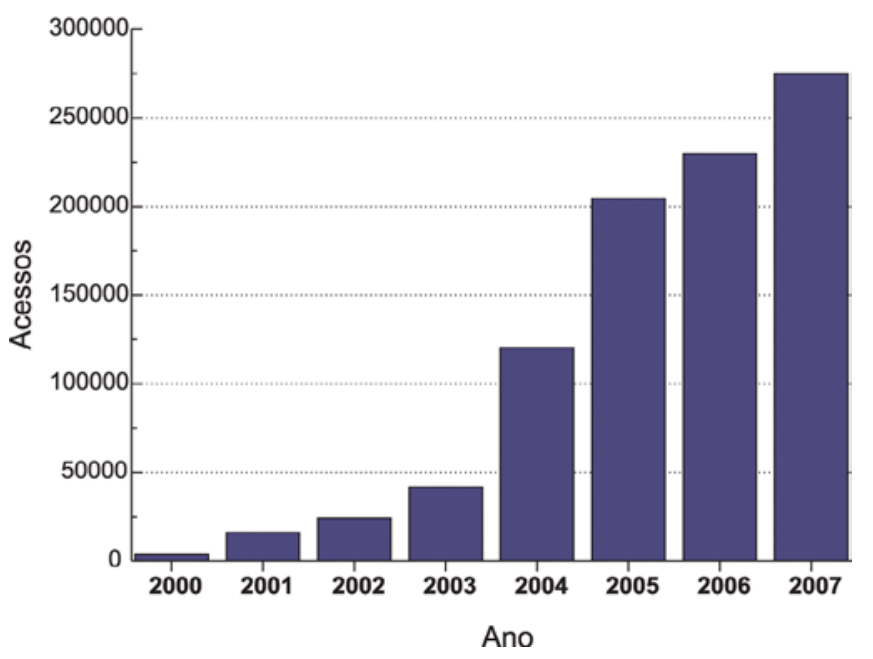

Figura 13. Total de acessos ao JBCS pelo SciELO (2002-2007)

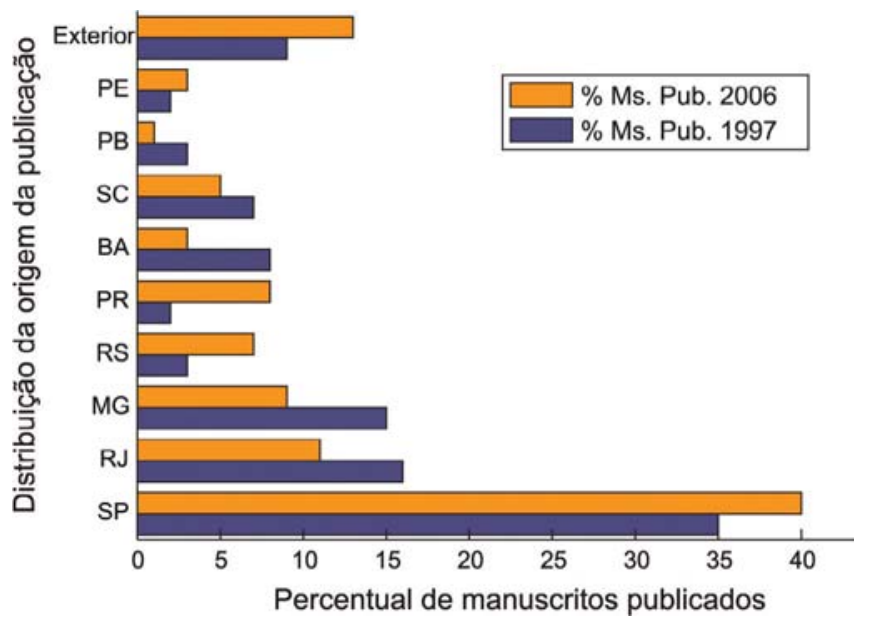

Figura 14. Distribuição de artigos nacionais publicados no JBCS (19972006)

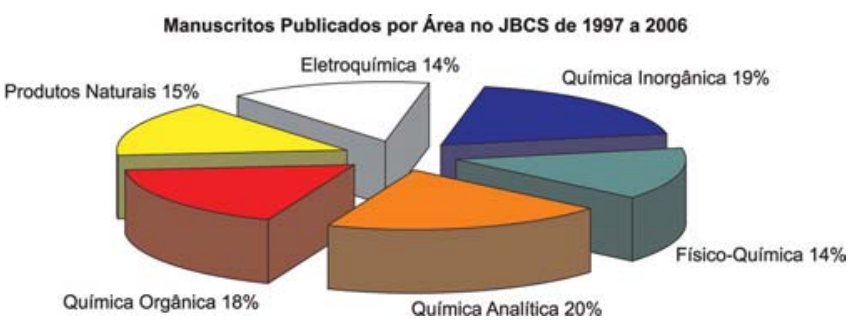

Figura 15. Distribuição por área de conhecimento dos manuscritos publicados no JBCS de 1997 a 2006

da no Web of Science é sobre reações de Diels-Alder catalisadas por ácidos de Lewis quirais ${ }^{35}$. Das outras 3 revisões, 2 são do mesmo autor e abordam líquidos iônicos ${ }^{36,37}$. A terceira é sobre reações de transesterificação de óleos vegetais ${ }^{33}$ e antecipa em alguns anos a explosão de artigos publicados na literatura sobre a produção, propriedades e uso do biodiesel.

O $J B C S$ tem buscado ampliar o número de artigos e a sua abrangência continuamente. No período 2004-2007 cerca de 1/3 dos assessores consultados foram do exterior. Em 2005 foi publicado um número especial em conjunto com a Revista Mexicana de Quimica ${ }^{55}$ que aproximou as duas editorias, sociedades e as comunidades de química. Em 2006, o JBCS ampliou significativamente o número de artigos publicados, passando para oito números anuais, sendo dois destes especiais ${ }^{56,57}$.

\section{CONCLUSÕES}

A Química Nova e o Journal of the Brazilian Chemical Society são dois exemplos bem sucedidos de ações da Sociedade Brasileira de Química e modelos que podem ser seguidos por sociedades científicas de outros países. Pilares da SBQ, estas duas revistas são demonstrações inequívocas que idealismo, utopia e dignidade são ingredientes necessários para transformação de sonhos em realidade. Poucos foram os acreditaram que a comunidade química brasileira viria a ter, como tem hoje, dois periódicos científicos indexados

Tabela 5. Artigos mais citados no JBCS desde sua criação*

\begin{tabular}{|c|c|c|c|c|}
\hline Artigo & $\begin{array}{l}\text { J. Braz. Chem. Soc. } \\
\text { Ano, Volume, Pgs. }\end{array}$ & Autores & Categoria & № Citações \\
\hline 1 & $\mathbf{1 9 9 7}, 8,289-332$ & Dias, L.C. & Revisão & $125^{35}$ \\
\hline 2 & $\mathbf{2 0 0 0}, 11,337-344$ & Dupont, J.; Consorti, C.S.; Spencer, J. & Revisão & $84^{36}$ \\
\hline 3 & $\mathbf{2 0 0 4}, 15,341-350$ & Dupont, J. & Revisão & $81^{37}$ \\
\hline 4 & $1998,9,199-210$ & Schuchardt, U.; Sercheli, R.; Vargas, R.M. & Revisão & $73^{33}$ \\
\hline 5 & $1998,9,435-440$ & Longhinotti, E.; Pozza, F.; Furlan, L. et al. & Artigo & $41^{38}$ \\
\hline 6 & $\mathbf{2 0 0 1}, 12,273-324$ & da Silva, J.F.M.; Garden, S.J.; Pinto, A.C. & Revisão & $35^{39}$ \\
\hline 7 & 2001, $12,159-164$ & Foschiera, J.L.; Pizzolato, T.M.; Benvenutti, E.V. & Artigo & $31^{40}$ \\
\hline 8 & 1998, $9,494-498$ & Gomes, L.A.D.; Padilha, P.D.; Moreira, J.C. et al. & Artigo & $29^{41}$ \\
\hline 9 & 2000, 11, 293-297 & Dupont, J.; Suarez, P.A.Z.; Umpierre, A.P. et al. & Artigo & $23^{42}$ \\
\hline 10 & $1999,10,241-262$ & Gunther, H. & Revisão & $23^{43}$ \\
\hline 11 & $\mathbf{1 9 9 8}, 9,219-223$ & de Andrade, J.B.; Andrade, M.V.; Pinheiro, H.L.C. & Artigo & $23^{44}$ \\
\hline 12 & $1995,6,267-270$ & Toma, H.E.; Alexiou, A.D.P. & Artigo & $23^{45}$ \\
\hline 13 & $\mathbf{2 0 0 3}, 14,198-219$ & Pasquini, C. & Revisão & $22^{46}$ \\
\hline 14 & $\mathbf{2 0 0 1}, 12,325-338$ & De Moura, K.C.G.; Emery, F.S.; Neves-Pinto. C. et al. & Artigo & $22^{47}$ \\
\hline 15 & 2001, 12, 695-705 & Gushikem, Y.; Rosatto, S.S. & Revisão & $21^{48}$ \\
\hline 16 & $\mathbf{1 9 9 8}, 9,273-278$ & Gushikem, Y.; Campos, E.A. & Artigo & $21^{49}$ \\
\hline 17 & $\mathbf{1 9 9 5}, 6,291-296$ & Cestari, A.R.; Airoldi, C. & Artigo & $21^{50}$ \\
\hline 18 & $\mathbf{2 0 0 3}, 14,230-243$ & Freire, R.S.; Pessoa, C.A.; Mello, L,D. et al. & Revisão & $20^{51}$ \\
\hline 19 & $2000,11,32-38$ & Varela, H.; Torresi, R.M.; Buttry, D.A. & Artigo & $20^{52}$ \\
\hline 20 & $1997,8,479-485$ & Martelli, P.B.; Reis, B.F.; Korn, M. et al. & Artigo & $20^{53}$ \\
\hline 21 & 1996, $7,19-30$ & Cardoso, S.L.; Nicodem, D.E.; Moore, T.A. et al. & Artigo & $20^{54}$ \\
\hline
\end{tabular}

* Fonte: Web of Science (http://portal.isiknowledge.com). Data de atualização: 21/09/2007 
aos principais bancos de dados internacionais. Permanecer na vitrine e continuar a crescer com qualidade são desafios permanentes. Vencê-los exige desprendimento, transpiração diária e uma boa dose de utopia. Mas, como disse Guimarães Rosa, viver é muito perigoso. Perigo maior é viver tendo que manter a periodicidade e a qualidade de Química Nova e do JBCS.

\section{AGRADECIMENTOS}

Ao Corpo Editorial, Corpo Técnico, Revisores e em especial aos Autores que tornaram possível a criação e a consolidação da qualidade editorial de $Q N$ e $J B C S$. Agradecemos também ao CNPq, à CAPES e FAPESP pelo inestimável apoio financeiro.

\section{REFERENCIAS}

1. Galembeck, F.; Edição Comemorativa dos 10 Anos da SBQ 1997, julho, 18.

2. Roth, D. L.; Curr. Sci. 2005, 89, 1531.

3. Marques, F.; Pesquisa FAPESP 2006, $n^{\circ} 126,24$.

4. Benchimol, J. L.; Cerqueira, R. C.; Martins, R. B.; Mendonça, A.; História, Ciências, Saúde - Manguinhos 2007, 14, 1.

5. Maciel, M. A. M.; Pinto, A. C.; Veiga Jr., V. F.; Quim. Nova 2002, 25, 429.

6. Veiga Jr.; V. F., Pinto; A. C.; Maciel, M. A. M.; Quim. Nova 2005, 28, 519

7. Pinto, A. C.; Silva, D. H. S.; Bolzani, V. S.; Lopes, N. P.; Epifanio, R. A.; Quim. Nova 2002, 25, 45.

8. Vidotti, E. C.; Rollemberg, M. C. E.; Quim. Nova 2004, 27, 139.

9. Castro, H. F.; Mendes, A. A.; Santos, J. C.; Quim. Nova 2004, 27, 146.

10. Costa Neto, P. R.; Rossi, L. F. S.; Zagonel, G. F.; Ramos, L. P.; Quim. Nova 2000, 23, 531 .

11. Ferrari, R. A.; Oliveira, V. S.; Scabio, A.; Quim. Nova 2005, 28, 19

12. Eichler, M.; Del Pino, J. C.; Quim. Nova 2000, 23, 835.

13. Santos, A. R.; Vidotti, E. C.; Silva, E. L.; Maionchi, F.; Hioka, N.; Quim. Nova 2002, 25, 844.

14. Terci, D. B. L.; Rossi, A. V.; Quim. Nova 2002, 25, 684.

15. Ferreira, M. M. C.; Antunes, A. M.; Melgo, M. S.; Volpe, P. L. O.; Quim. Nova 1999, 22, 724

16. Mattoso, L. H. C.; Quim. Nova 1996, 19, 388

17. Moreira, J. C.; Barek, K.; Quim. Nova 1995, 18, 362.

18. Guaratini, C. C. I.; Zanoni, M. V. B.; Ouim. Nova 2000, 23,71

19. Lopes,W. A.; de Andrade, J. B.; Quim. Nova 1996, 19, 497.

20. Arakaki, L. N. H.; Airoldi, C.; Quim. Nova 1999, 22, 246.

21. Coelho, F.; Almeida, W. P.; Quim. Nova 2000, 23, 98.

22. Crepaldi, E. L.; Valim, J. B.; Quim. Nova 1998, 21, 300.

23. Aleixo, P. C.; Nóbrega, J. A.; Santos Jr., D.; Muller, R. C. S.; Quim. Nova 2000, 23, 310 .
24. Maia, D. J.; De Paoli, M-A.; Alves, O. L.; Zarbin, A. J. G.; das Neves, S.; Quim. Nova 2000, 23, 204.

25. Pinto, A. C.; de Andrade, J. B.; Quim. Nova 1999, 22, 448.

26. Ferraz, H. M. C.; Pardini, V. L.; Quim. Nova 1997, 20 (Especial), 75.

27. de Castro, L. A. B.; Prescott, E.; Quim. Nova 1997, 20 (Especial), 15.

28. Paniago, E. B.; Quim. Nova 1997, 20 (Especial), 23.

29. Pinto, A. C.; Peixoto, E. A. M.; Galembeck, F.; de Andrade, J. B.; de Paoli, M. A.; Avaca, L. A.; Alves, O. L.; Quim. Nova 1997, 20 (Especial), 81.

30. Loh, W.; Vargas, M. D.; J. Braz. Chem. Soc. 2005, 16, 3.

31. Loh, W.; Dias, L. C.; J. Braz. Chem. Soc. 2006, 17, 3.

32. Loh, W.; Dias, L. C.; J. Braz. Chem. Soc. 2007, 18, 3.

33. Schuchardt, U.; Sercheli, R.; Vargas, R. M.; J. Braz. Chem. Soc. 1998, 9, 199.

34. Pinto, A. C.; Guarieiro, L. L. N.; Rezende, M. J. C.; Ribeiro, N. M.; Torres, E. A.; Lopes, W. A.; Pereira, P. A. P.; de Andrade, J. B.; J. Braz. Chem. Soc. 2005, 16, 1313 .

35. Dias, L. C.; J. Braz. Chem. Soc. 1997, 8, 289.

36. Dupont, J.; Consorti, C. S.; Spencer, J.; J. Braz. Chem. Soc. 2000, 11, 337

37. Dupont, J.; J. Braz. Chem. Soc. 2004, 15, 341.

38. Longhinotti, E.; Pozza, F.; Furlan, L.; Sanchez, M. N. M.; Klug, M. Laranjeira, M. C. M.; Fávere, V. T.; J. Braz. Chem. Soc. 1998, 9, 435.

39. da Silva, J. F. M.; Garden, S. J.; Pinto, A. C.; J. Braz. Chem. Soc. 2001, 12, 273.

40. Foschiera, J. L.; Pizzolato, T. M.; Benvenutti, E. V.; J. Braz. Chem. Soc. 2001, 12, 159.

41. Gomes, L. A. D., Padilha, P. D., Moreira, J. C.; Newton, L.; Dias Filho, N. L.; Gushikem, Y.; J. Braz. Chem. Soc. 1998, 9, 494.

42. Dupont, J.; Suarez, P. A. Z.; Umpierre, A. P., de Souza, R. F.; J. Braz. Chem. Soc. 2000, 11, 293.

43. Gunther, H.; J. Braz. Chem. Soc. 1999, 10, 241.

44. de Andrade, J. B.; Andrade, M. V.; Pinheiro, H. L. C.; J. Braz. Chem. Soc. 1998, 9, 219.

45. Toma, H. E.; Alexiou, A. D. P.; J. Braz. Chem. Soc. 1995, 6, 267.

46. Pasquini, C.; J. Braz. Chem. Soc. 2003, 14, 198.

47. De Moura, K. C. G.; Emery, F. S.; Neves-Pinto, C.; Pinto, M. C. F. R.; Dantas, A. P.; Salomão, K.; de Castro, S. L.; Pinto, A. V.; J. Braz. Chem. Soc. 2001, 12, 325.

48. Gushikem, Y., Rosatto, S. S.; J. Braz. Chem. Soc. 2001, 12, 695.

49. Gushikem, Y., Campos, E. A.; J. Braz. Chem. Soc. 1998, 9, 273.

50. Cestari, A. R., Airoldi, C.; J. Braz. Chem. Soc. 1995, 6, 291.

51. Freire, R. S.; Pessoa, C. A.; Mello, L. D., Kubota, L. T.; J. Braz. Chem. Soc. 2003, 14, 230.

52. Varela, H.; Torresi, R. M.; Buttry. D. A.; J. Braz. Chem. Soc. 2000, 11, 32

53. Martelli, P. B.; Reis, B. F.; Korn, M.; Rufini, I. A.; J. Braz. Chem. Soc 1997, 8, 479.

54. Cardoso, S. L.; Nicodem, D. E.; Moore, T. A.; Moore, A. L.; Gust, D.; J. Braz. Chem. Soc. 1996, 7, 19.

55. Delgado, G.; Torresi, R. M.; J. Braz. Chem. Soc. 2005, 16, 289

56. de Castro, L. D.; J. Braz. Chem. Soc. 2006, 17, 1053.

57. Toma, H. E.; J. Braz. Chem. Soc. 2006, 17, 1457. 